\title{
Electronic structure of atomically coherent square semiconductor superlattices with dimensionality below two
}

\author{
E. Kalesaki, ${ }^{1,2}$ W. H. Evers,${ }^{3,4}$ G. Allan, ${ }^{1}$ D. Vanmaekelbergh,,${ }^{5}$ and C. Delerue ${ }^{1, *}$ \\ ${ }^{1}$ IEMN - Department ISEN, UMR CNRS 8520, Lille, France \\ ${ }^{2}$ Physics and Materials Science Research Unit, University of Luxembourg, 162 a avenue de la Faïencerie, L-1511 Luxembourg \\ ${ }^{3}$ Opto-electronic Materials section, Chemical Engineering, Delft University of Technology, 2628 BL, The Netherlands \\ ${ }^{4}$ Kavli Institute of Nanoscience, Delft University of Technology, 2628 CJ, The Netherlands \\ ${ }^{5}$ Debye Institute for NanoMaterials Science, Utrecht University, Princetonplein 1, NL-3508 TH Utrecht, The Netherlands
}

(Received 18 July 2013; published 25 September 2013)

\begin{abstract}
The electronic structure of recently synthesized square superlattices with atomic coherence composed of PbSe, $\mathrm{CdSe}$, or CdTe nanocrystals (NCs) attached along $\{100\}$ facets is investigated using tight-binding calculations. In experimental realizations of these systems [W. H. Evers et al., Nano Lett. 13, 2317 (2013)], NC facets are atomically bonded, resulting in single-crystalline sheets, which, due to their nanogeometry, have an effective dimensionality below two. We predict electronic structures composed of successive bands formed by strong coupling between the wave functions of nearest-neighbor NCs. This coupling is mainly determined by the number of atoms at the NC bonding plane. The band structures deviate markedly from that of the corresponding two-dimensional (2D) quantum well; the 2D case can be recovered, however, if the effects of the nanogeometry are gradually reduced. The width of the bands can reach hundreds of $\mathrm{meV}$, ascribing highly promising transport properties to square superlattices. The band edges are located at $k=0$ except for PbSe superlattices, where their position in $k$ space surprisingly depends on the parity of the number of $\{100\}$ atomic planes in the NCs. Our calculations demonstrate that semiconductors with dimensionality below two have a strong potential for (opto-)electronic, photovoltaic, and spintronic applications.
\end{abstract}

DOI: 10.1103/PhysRevB.88.115431

PACS number(s): 73.22.-f, 62.23.Kn, 81.07.-b, 73.21.Cd

\section{INTRODUCTION}

\section{A. Two-dimensional semiconductors}

Two-dimensional (2D) semiconductors have been extensively investigated during the past few decades due to their wide variety of applications in transistors, solar cells, photodetectors, light emitting diodes, and lasers [e.g., Ref. 1 and references therein]. Recently the scientific interest in 2D systems, in which carriers experience confinement in one dimension but are allowed to move freely along the other two directions, has experienced a considerable rise owing mainly to the emergence of graphene ${ }^{2}$ and topological insulators. ${ }^{3,4}$ Up to date, most experimental studies on crystalline $2 \mathrm{D}$ semiconductors have focused on III-V and II-VI materials grown by gas phase methods in high vacuum, such as molecular beam epitaxy. ${ }^{5}$ These approaches have been extremely successful but their technological applications are often limited by their high cost and technical requirements. In addition, the presence of strain between the film and substrate materials significantly affects their electronic properties and may hinder exploitation of their full potential. These impediments could be surpassed by recent achievements in wet-chemical semiconductor fabrication based on nanocrystalline colloids. This is exemplified, for instance, by the synthesis of remarkable 2D Cd-chalcogenide platelets (suspended in solution) with a thickness controlled at the atomic level. 6,7

\section{B. Square superlattices}

In the present paper we consider planar semiconductor sheets realized by facet-specific oriented attachment of colloidal nanocrystals (NCs), a new approach recently reported by Evers et al. ${ }^{8}$ Due to atomic bonding of particular facets and the specific truncated nanocube shape of the building blocks, the resulting planar sheets show a square nanogeometry, i.e., a square periodic structure of holes, and will be hereafter called square superlattices. Examples of square superlattices of $\mathrm{PbSe}$ and $\mathrm{CdSe}$ are presented in Fig. 1. We should remark here that these systems are atomically coherent and thus show strong electronic coupling between the building blocks. ${ }^{8}$ Quite similar superlattices with square nanogeometry were recently reported by another group. ${ }^{9}$ The calculations below will show that the band structure deviates from that of genuine 2D semiconductors, and that these systems in fact correspond to a dimensionality that can gradually decrease below 2, depending on the nanogeometry. The synthesis of square superlattices comprising rock-salt $\mathrm{PbSe} \mathrm{NCs}$ was accomplished through NC self-assembly and facet-specific atomic attachment. Conversion of the rock-salt $\mathrm{PbSe}$ square superlattices into zinc-blende $\mathrm{CdSe}$ by a $\mathrm{Cd}$-for- $\mathrm{Pb}$ cation exchange has also been demonstrated. ${ }^{8}$ With the same procedure, formation of $2 \mathrm{D} \mathrm{PbTe}(\mathrm{CdTe})$ superlattices is plausible. ${ }^{10}$ We should remark here that in these systems the contact region between two nanocrystals is formed by the defect-free lattice itself; hence there is no electronic barrier between the nanocrystals in the system. This is different from the well-studied self-assembled nanocrystal superlattices in which the nanocrystals are separated by an organic or inorganic barrier, resulting in a much weaker coupling. ${ }^{11-16}$

The strict and commonly used classification of semiconductors into $0 \mathrm{D}$ quantum dots, $1 \mathrm{D}$ rods, and 2D quantum wells has overlooked the remarkable effects of a more complex nanogeometry on the electronic structure. In the present case of square superlattices, this electronic structure is also determined by the nanogeometry, i.e., the periodic array of holes in 

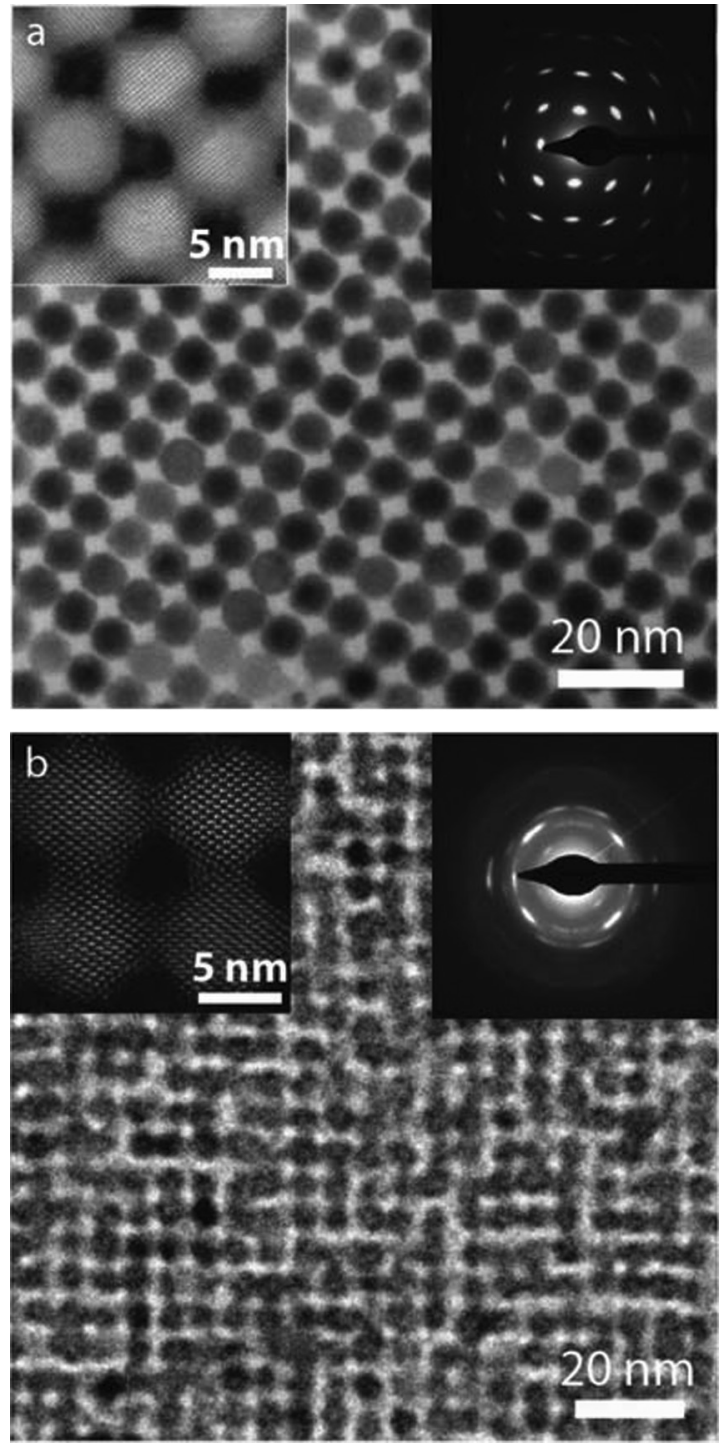

FIG. 1. TEM images of (a) PbSe and (b) CdSe square lattices consisting of atomically connected semiconductor truncated nanocubes with a size of $6 \mathrm{~nm}$. In the left insets HAADF-STEM images along the $\langle 100\rangle$ axis are shown, clearly indicating the atomically defined crystalline connection between the NCs. The right insets are electrodiffractograms of an area $>1 \mu \mathrm{m}^{2}$ demonstrating the long-range atomic order of the crystal.

the planar structure, which depends on the size and shape of the building blocks used in the self-assembly. Besides the nanogeometry, the atomic structure of the semiconductor itself (zinc-blende for $\mathrm{CdSe}$ and $\mathrm{CdTe}$, rock-salt for $\mathrm{PbSe}$ ) is important. That both the nanogeometry and the atomic lattice are important in the electronic structure has also been demonstrated in our recent study of artificial graphene superlattices of $\mathrm{PbSe}, \mathrm{CdSe}$, and $\mathrm{CdTe} \mathrm{NCs}^{10}$ obtained by the same kind of synthesis. ${ }^{8}$

In bulk, $\mathrm{CdSe}$ and CdTe have a direct gap above $1.5 \mathrm{eV}$ at the $\Gamma$ point of the Brillouin zone. In contrast, bulk PbSe has a small gap of $280 \mathrm{meV}$ at $300 \mathrm{~K}^{17}$ at the $L$ point of the Brillouin zone, which makes it interesting for near-infrared optoelectronics applications. From a theoretical viewpoint, the fundamental properties of CdSe and CdTe NCs have been studied for several decades, ${ }^{18-23}$ mostly for NCs with a wurtzite lattice. PbSe has come under the spotlight of scientific research more recently, with precedent investigations focusing mainly on the electronic and optical properties of individual NCs. ${ }^{24-32}$

\section{METHODOLOGY}

\section{A. Tight-binding calculations}

In our current contribution, the electronic structure of square superlattices of $\mathrm{PbSe}, \mathrm{CdSe}$, and $\mathrm{CdTe} \mathrm{NCs}$ is explored through tight-binding calculations, which allows us to treat system sizes accessible to experiments. Each atom in the superlattice $(\mathrm{Pb}, \mathrm{Cd}, \mathrm{Se}$, or $\mathrm{Te})$ is described by a double set of $s p^{3} d^{5} s^{\star}$ atomic orbitals including the spin degree of freedom. Implementation of a $s p^{3} d^{5} s^{\star}$ basis results in the accurate description of the bulk band structures in comparison with ab initio calculations and available experimental data, ${ }^{18,24}$ ensuring transferability of the parameters for investigating the electronic structure of semiconductor nanostructures. ${ }^{33}$ Presently used tight-binding parameters for $\mathrm{PbSe}$ are taken from Ref. 24, those for zinc-blende CdSe and CdTe are given in Appendix A. Spin-orbit coupling is included. In the case of zinc-blende CdSe and CdTe superlattices, surfaces are saturated by pseudohydrogen atoms in order to avoid states induced in the band gap due to surface dangling bonds. For rock-salt $\mathrm{PbSe}$ nanostructures, surface passivation is not necessary. ${ }^{24}$ Due to the large size of the systems considered (up to $15 \times 10^{3}$ atoms and $3 \times 10^{5}$ atomic orbitals per unit cell), the numerical methods described by Niquet et $a l^{34}$ are used to calculate near-gap eigenstates.

\section{B. Geometry of the superlattices}

Square superlattices are modeled in accordance with the experimental realizations of Evers et al., ${ }^{8}$ i.e., 2D lattices of $\langle 001\rangle$-oriented $\mathrm{PbSe}, \mathrm{CdSe}$, or $\mathrm{CdTe} \mathrm{NCs}$ attached via perpendicular $\{100\}$ facets (Figs. 1 and 2). Each NC has the form of a truncated nanocube, comprising $6\{100\}, 8\{111\}$, and $12\{110\}$ facets. The positions of the vertices of the NC shape are given by $P[ \pm 1, \pm(1-q), \pm(1-q)]$, where $[ \pm 1, \pm 1, \pm 1]$ indicate the position of the six corners of the original nanocube $(q=0), q \leqslant 1$ is the truncation factor, and $P$ represents all possible permutations. Realistic NC shapes are considered, having truncations $q$ between 0.25 and $0.5{ }^{8}$ The system unit cell includes a single NC, attached to its periodic images along two orthogonal $\langle 100\rangle$ directions. The NC size is defined by the number of atomic biplanes along $\langle 100\rangle$ directions and the length of the vectors delineating the superlattice is $n a$ where $n$ is an integer and $a$ is the lattice parameter $(0.612 \mathrm{~nm}$ for $\mathrm{PbSe}, 0.608 \mathrm{~nm}$ for CdSe, and $0.648 \mathrm{~nm}$ for $\mathrm{CdTe}$ ). NC sizes ranging from 2.4 to $7.4 \mathrm{~nm}$ were assessed for all materials considered.

\section{RESULTS FOR SUPERLATTICES OF CdSe}

\section{A. Band structure: Conduction bands}

First, we consider square superlattices of zinc-blende CdSe (CdTe) as these systems have a simpler band structure of the bulk materials compared to rock-salt PbSe. In Figs. 3(a) and 3(d) the electronic structure of a CdSe NC superlattice without truncation $(q=0)$, i.e., a uniform 2D film, is presented and 

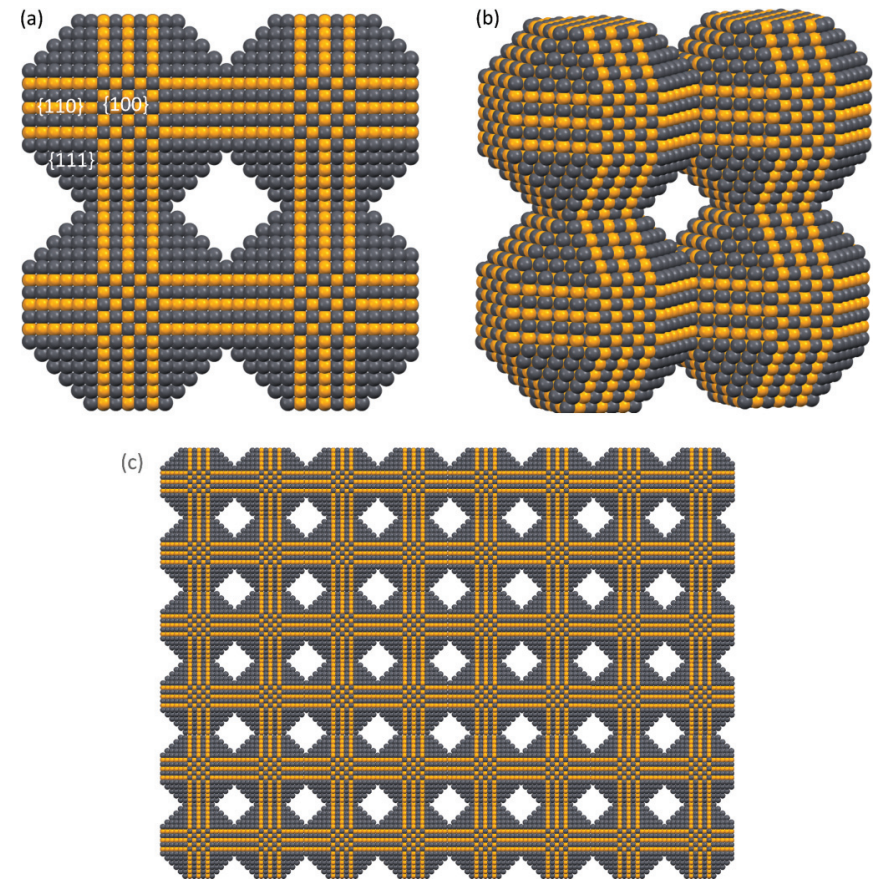

FIG. 2. (Color online) Model structure of colloidal PbSe NCs, oriented along $\langle 100\rangle$ and attached via perpendicular $\{100\}$ facets, resulting in a square superlattice. An ensemble of four truncated nanocubes is illustrated in the top view along $\langle 001\rangle$ (a) and tilted relative to $\langle 001\rangle$ (b) to better visualize the nanocube shape and attachment. The NCs present $6\{100\}, 8\{111\}$, and $12\{110\}$ facets. Gray/dark colored (gold/light colored) spheres are used to indicate $\mathrm{Pb}(\mathrm{Se})$ atoms, respectively. In (c) part of a square superlattice formed by $\mathrm{PbSe} \mathrm{NCs}$ is presented in the top view.

compared with the corresponding electronic structure of a NC superlattice with $q=0.45$ [Figs. 3(b) and 3(e)] as well as that of individual NCs with the same truncation [Figs. 3(c) and 3(f)]. For the sake of comparison, all cases refer to the same
NC size and band structures are presented in the Brillouin zone of the superlattice with $q=0.45$.

The electronic structure of the uniform film $(q=0)$ is characterized by bands which are very dispersive on a wide energy range because carriers are totally free to move in two directions. The bands are just folded back in the Brillouin zone of the superlattice (see also Appendix B). When the nanocubes are truncated, assembly results in a planar quasi-2D system with a periodic array of holes. In that case, the electronic structure is characterized by multiple bands, as expected for superlattices. ${ }^{35}$ The truncations induce periodic scattering of the electronic waves, opening gaps in particular at the center and at the edges of the superlattice Brillouin zone, exactly like when we consider quasifree electron bands starting from free electron ones. ${ }^{33,36}$ We show in Appendix B that the conduction band structure of the square superlattices can be easily deduced from the case of the uniform film if we define pseudopotentials which describe the effects of the truncations on the electron waves.

On the other hand, results on the superlattices of truncated NCs can be also interpreted starting from the limit of individual NCs [Figs. 3(c) and 3(f)]. Each individual NC is characterized by discrete energy levels as a consequence of the strong quantum confinement. Facet-to-facet atomic bonding of the NCs induces strong coupling between the wave functions of first-nearest neighbor NCs and the system can be perceived as a single quasi-2D crystal yet with a superimposed nanogeometry. The bands are formed exactly like bands in solids that are strictly $2 \mathrm{D}$, arising from the coupling between atomic orbitals.

\section{B. Effective tight-binding model}

In an alternative and more coarse fashion, the building blocks of the square superlattices can also be seen as "artificial" atoms. The conduction bands (CBs) can then be described using a simple effective tight-binding Hamiltonian characterized
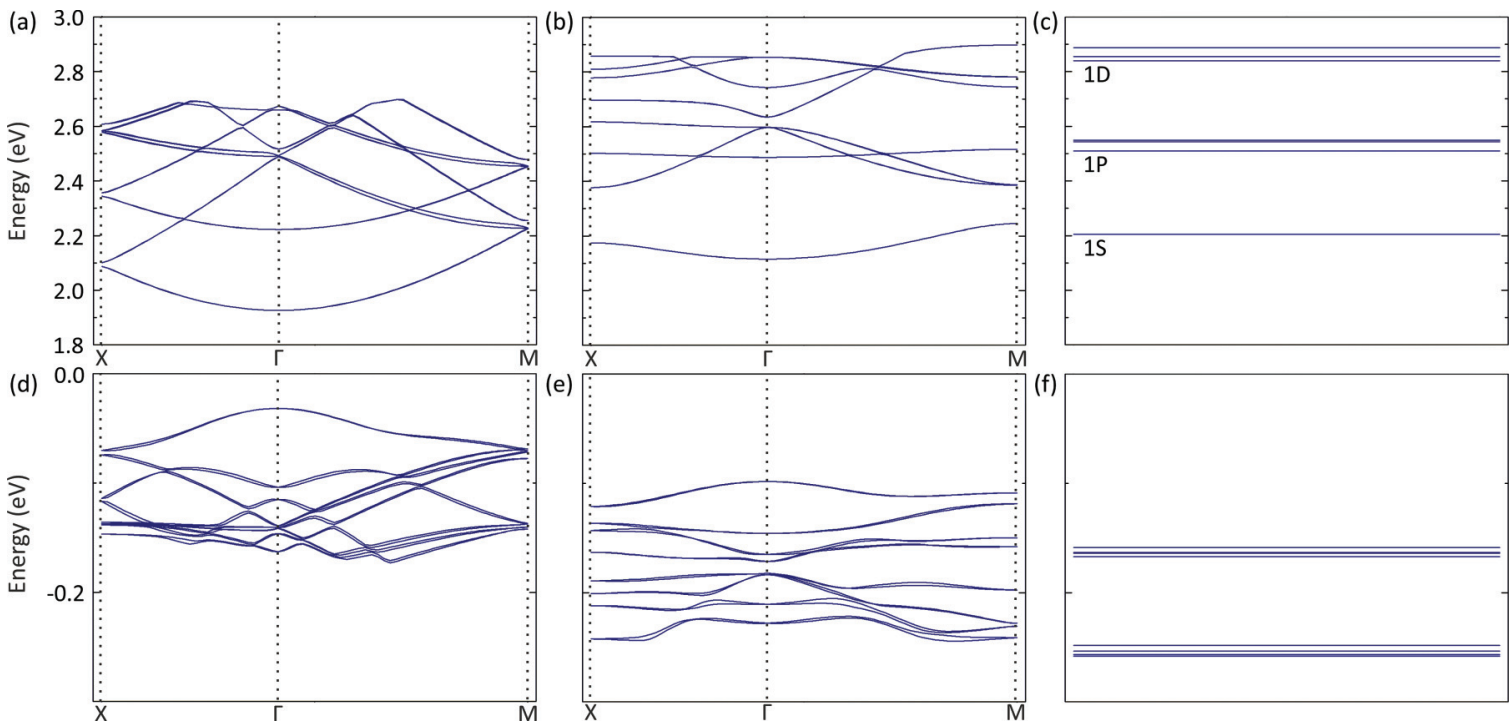

FIG. 3. (Color online) (a) Conduction and (d) valence band structure for a superlattice of CdSe NCs with zero truncation, i.e., a uniform 2D film. (b) and (e) Corresponding plots for a square superlattice of truncated NCs $(q=0.45)$. (c) and (f) Equivalent plots for isolated CdSe NCs $(q=0.45)$. The same NC size is considered in all cases, i.e., $4.26 \mathrm{~nm}$ along $\langle 100\rangle$. Sixteen valence bands and 16 conduction bands are depicted. Conduction levels of the isolated NCs are labeled according to the symmetry of the envelope wave function $(1 S, 1 P, 1 D)$. 

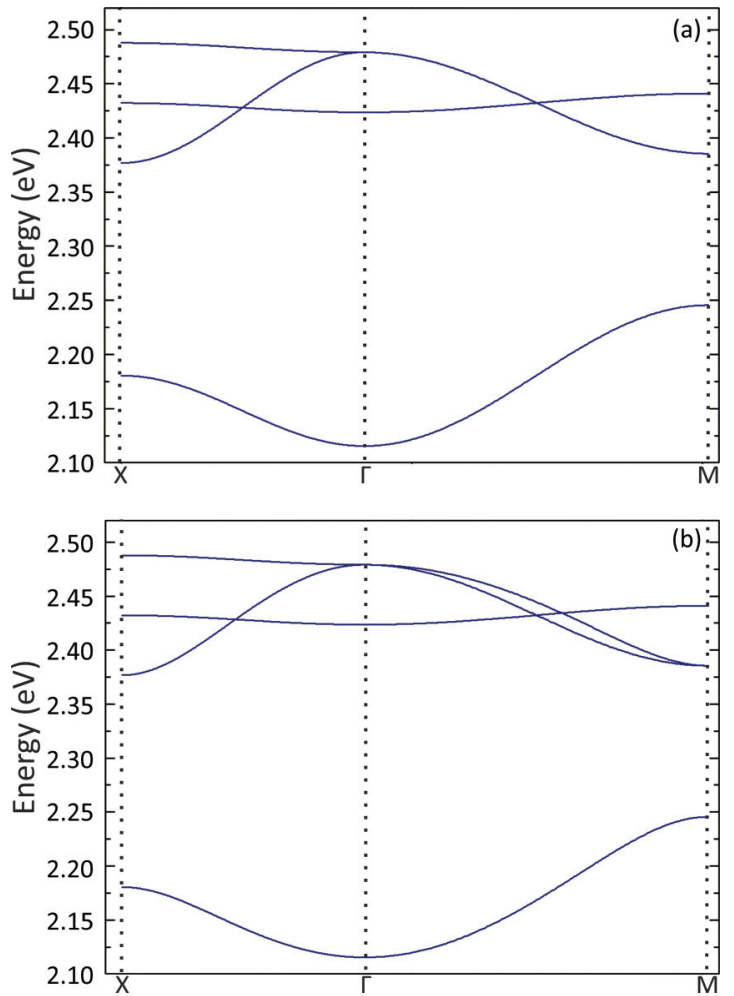

FIG. 4. (Color online) Conduction band structure of a square superlattice of CdSe NCs using the effective tight-binding Hamiltonian for two sets of parameters: (a) $E_{s}=2.18 \mathrm{eV}, E_{p}=2.43 \mathrm{eV}, V_{s s \sigma}=$ $-16 \mathrm{meV}, V_{s p \sigma}=0 \mathrm{meV}, V_{p p \sigma}=26 \mathrm{meV}$, and $V_{p p \pi}=-2.2 \mathrm{meV}$. (b) Same but with $V_{s p \sigma}=20 \mathrm{meV}$.

by a spin-degenerate electron state with a $1 S$ envelope wave function and by three spin-degenerate $1 P$ excited states higher in energy (Fig. 4). In this context, the on-site energies are $E_{S}$ and $E_{p}$. All hopping terms, i.e., nearest-neighbor interactions, can be written in the two-center approximation as functions of four parameters $\left(V_{s s \sigma}, V_{s p \sigma}, V_{p p \sigma}, V_{p p \pi}\right)$ plus geometrical factors, following Slater and Koster. ${ }^{37}$ It should be noted that $1 S$ and $1 P$ bands can be separately treated if $V_{s p \sigma}=0$.

Using reasonable parameters, this simplified scheme explains quite well the results of $s p^{3} d^{5} s^{\star}$ tight-binding calculations [Fig. 3(b)]. The lowest CB stems mainly from the $1 S$ wave functions of the NCs, while the bands higher in energy originate mostly from the $1 P$ states. Comparing Fig. 4 with full tight-binding calculations [Fig. 3(b)], it is evident that weak hybridization between $1 S$ and $1 P$ orbitals takes place in the superlattices, the agreement being better when $V_{s p \sigma} \neq 0$ [Fig. 4(b)]. In addition, the almost dispersionless bands observed under full tight-binding calculations at $\approx 2.5 \mathrm{eV}$ are due to weak coupling $\left(V_{p p \pi}\right)$ between $1 P_{z}$ states perpendicular to the lattice.

Based on the above, we conclude that the significant dispersion of the $1 S(1 P) \mathrm{CBs}$ in the uniform film is slightly reduced in $\mathrm{NC}$ superlattices due to the truncation which leads to a reduction in the number of atoms at the junction between neighbor NCs and enhanced scattering of the electronic waves. As a consequence, an opening of the gap between the $1 S$ and $1 P$ states in all high symmetry $k$ points is recorded as we pass from the uniform film to the square superlattice. The truncation also induces a vertical shift of the bands due to increased quantum confinement. We have found that each CB is approximately centered on the energy of the corresponding states of the isolated NCs. Moreover weak hybridization between $1 S$ and $1 P$ orbitals is evident for both $q \rightarrow 0$ and $q \neq 0$ cases.

\section{Band structure: Valence bands}

The above discussion is focused mainly on the CBs of CdSe NC superlattices since the valence bands (VBs) have a more complex behavior due to coupling between the anisotropic heavy-hole and light-hole bands induced by confinement. Hence their interpretation, especially starting from the limit of isolated NCs assembly is less trivial. However, an important
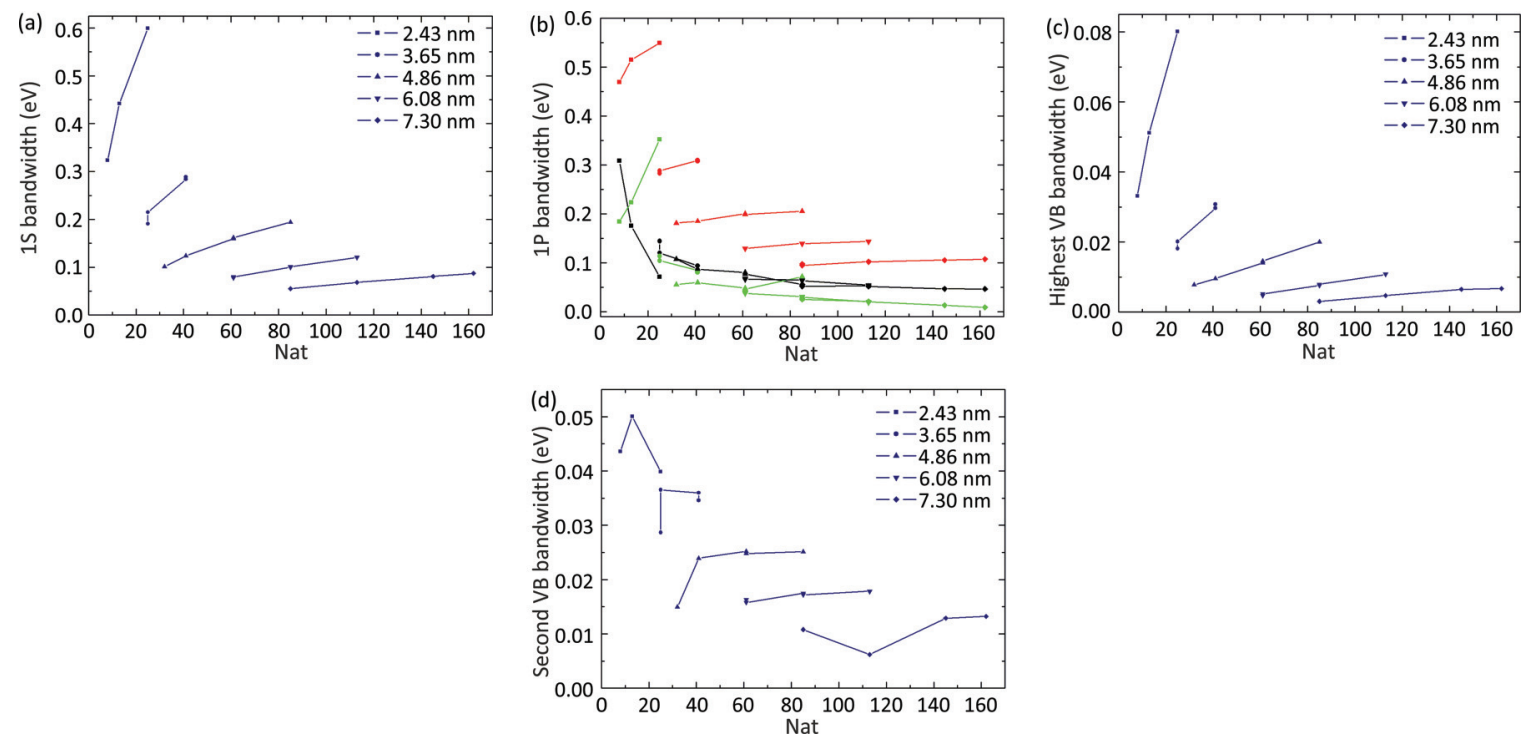

FIG. 5. (Color online) Bandwidth of the (a) $1 S$ and (b) $1 P$ CBs, (c) highest and (d) second VBs of square superlattices of CdSe NCs versus the number of atoms $\left(N_{\mathrm{at}}\right)$ at the NC bonding plane. In all cases NC size, indicated in the legends, increases from left to right. In (b) black, red, and green lines (darker to lighter color lines) are used to indicate the first, second, and third $1 P \mathrm{CB}$ states, respectively. 

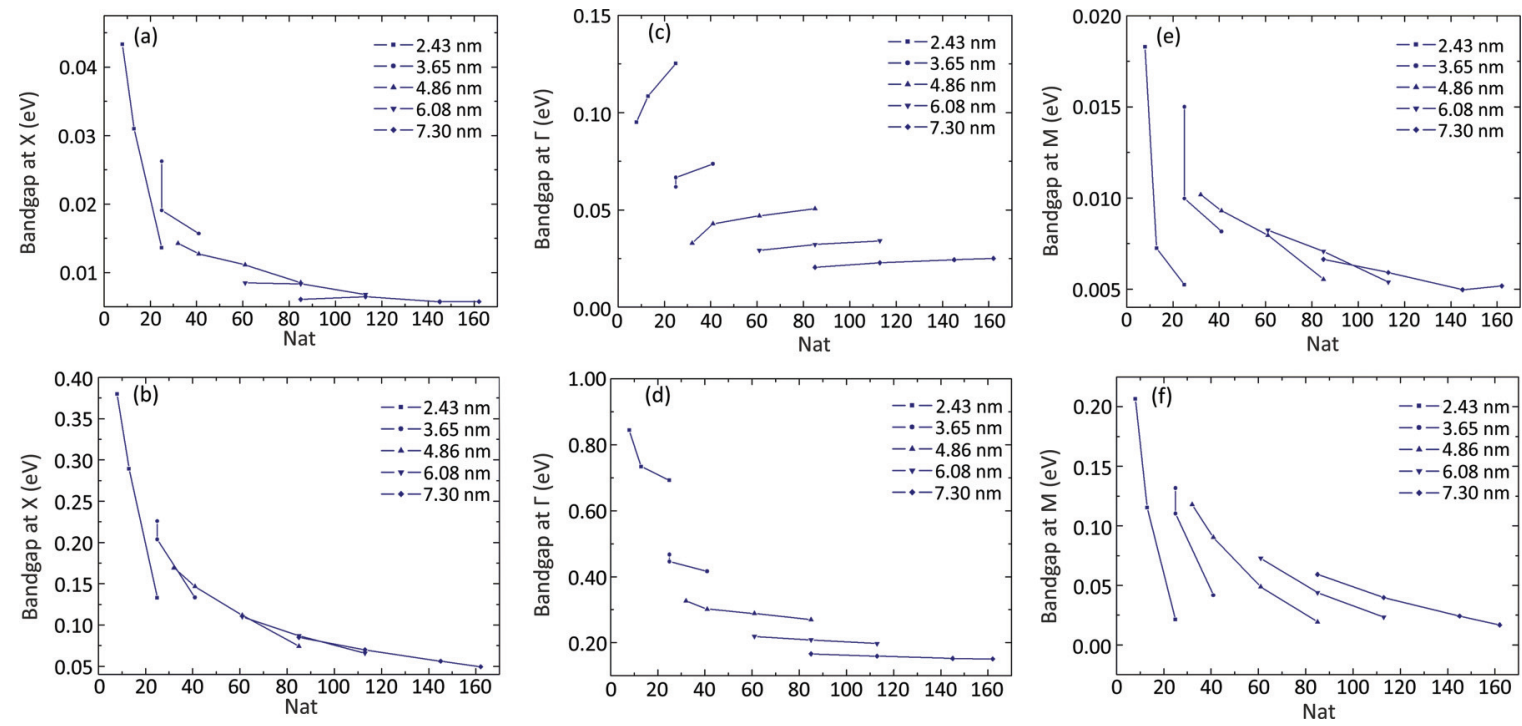

FIG. 6. (Color online) Energy gaps at $X, \Gamma$, and $M$ between the two highest VBs (a), (c), and (e) and between the two lowest CBs (b), (d), and (f) versus the number of atoms $\left(N_{\mathrm{at}}\right)$ at the $\mathrm{NC}$ bonding plane, for square superlattices of CdSe. In all cases NC size, indicated in the legends, increases from left to right.

feature revealed for both valence and conduction bands in square NC superlattices is their substantial dispersion, which indicates that bandlike transport of electrons as well as holes could be realized in such systems. Hence square superlattices are promising systems for photodetectors, solar cells, and field-effect transistors.

\section{Bandwidths and energy gaps}

The full bandwidths of $1 S$ and $1 P$ CBs are presented in Figs. 5(a) and 5(b) versus the number of atoms at the NC bonding plane. The NCs with the smallest truncation, having the maximum number of atoms at the $\mathrm{NC}$ bonding plane, exhibit in general maximum dispersions. The bandwidths of the topmost and second VBs [Figs. 5(c) and 5(d)] exhibit a similar trend but they are smaller due to heavier effective masses. The evolution of the energy gap between the two lowest CBs or between the two highest VBs at the high symmetry $k$ points $(X, \Gamma, M)$ is given in Fig. 6. Superlattices of NCs with the smallest truncation exhibit minimum gaps. Moreover maximum dispersions and gaps are recorded for the smallest size NC systems due to stronger confinement. Gap values in the VBs [Figs. 6(a), 6(c), and 6(e)] are always smaller than in the CBs [Figs. 6(b), 6(d), and 6(f)], as a consequence of bands derived from both heavy- and light-hole bands of bulk CdSe.

\section{RESULTS FOR SUPERLATTICES OF CdTe}

The electronic structure of square superlattices of CdTe NCs (Fig. 7) resembles that of CdSe. However, the strong intrinsic spin-orbit coupling in CdTe results in a considerable spin splitting not only in the VBs but also in the CBs, away from the high symmetry $k$ points of the Brillouin zone. This momentum-dependent splitting may be due to the combined effect of bulk inversion asymmetry (BIA) ${ }^{38}$ in the underlying zinc-blende lattice and asymmetry of the structure perpendicular to the superlattice, the so-called Rashba effect. ${ }^{39}$ Such large spin splittings are not a special effect of the nanogeometry but occur similarly pronounced in CdTe quantum wells ${ }^{40}$ and may be attractive for spintronic applications. ${ }^{41}$ The BIA term is induced by the lack of a center of inversion in zinc-blende binary semiconductors, ${ }^{38,42,43}$ whereas the structure inversion asymmetry term as already stated originates from the inversion asymmetry of the confining potential in, e.g., asymmetric quantum wells or bulk semiconductors under strain. ${ }^{39,41}$
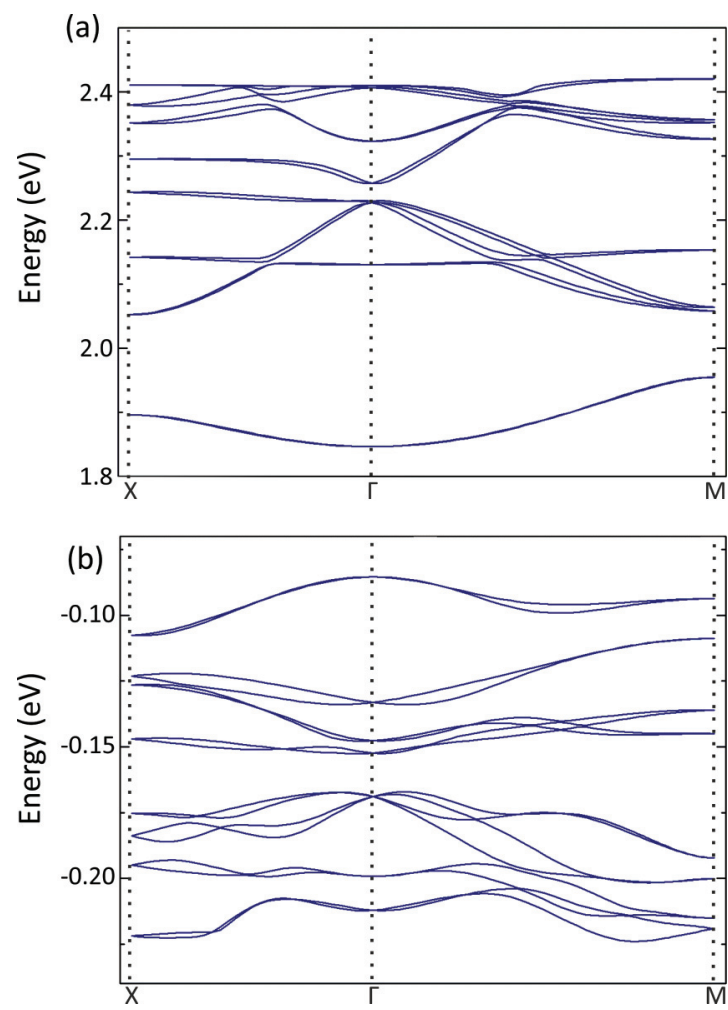

FIG. 7. (Color online) (a) Conduction and (b) valence band structure of a square superlattice of CdTe NCs $(\mathrm{NC}$ size $=4.54 \mathrm{~nm}$, $q=0.45)$. 


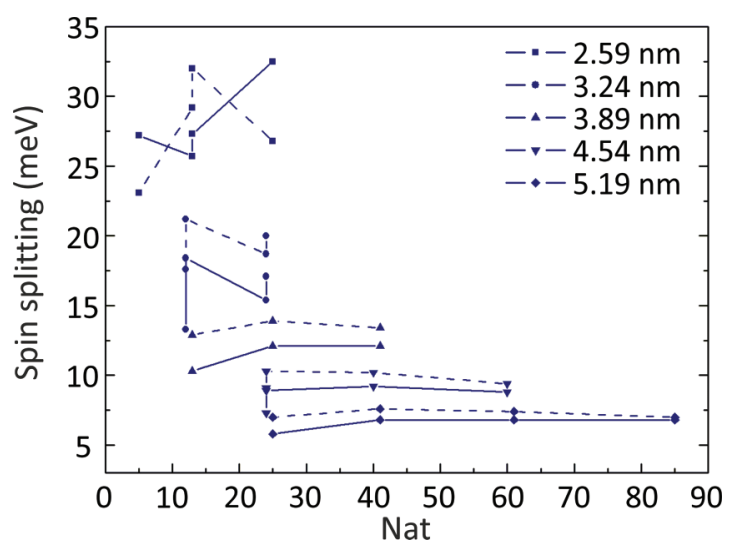

FIG. 8. (Color online) Spin splitting of the second $\mathrm{CB}$ at $\mathbf{k}=$ $0.5 \Gamma M$ for square superlattices of $\mathrm{CdTe} \mathrm{NCs}$ versus the number of atoms $\left(N_{\mathrm{at}}\right)$ at the $\mathrm{NC}$ bonding plane. $\mathrm{NC}$ sizes are indicated in the legend. Symmetric (solid lines) and asymmetric (dashed lines) superlattices are considered for comparison.

In order to identify the origin of the spin splitting in our systems, we have calculated the band structures for two types of superlattices, respectively, symmetric and asymmetric with respect to the $z$ axis. The asymmetric structure is just obtained from the symmetric one by removing the lowest plane of atoms. The spin splitting of the second CB at $\mathbf{k}=0.5 \Gamma M$ (where it is the largest) is displayed in Fig. 8 for the two configurations. It has a weak dependence on the number of atoms at the $\mathrm{NC}$ bonding plane but strongly increases at decreasing NC size. A large part of the spin splitting is already present in symmetric superlattices showing that it mainly comes from the BIA. The Rashba contribution, only present in the asymmetric case, is smaller but tends to increase for smaller size.

\section{RESULTS FOR SUPERLATTICES OF PbSe}

The case of square superlattices of rock-salt PbSe NCs is more complicated as evidenced in Fig. 9 where the band structures are presented for two NC sizes, $4.89 \mathrm{~nm}$ [Figs. 9(a) and $9(\mathrm{c})]$ and $4.28 \mathrm{~nm}$ [Figs. 9(b) and $9(\mathrm{~d})]$. However, there is clear mirror symmetry between CBs and VBs, even if it is not perfect. This is easily explained by a similar (approximate) symmetry of the bands in bulk $\mathrm{PbSe}^{24,27}$ In addition, the overall behavior of the lowest CBs shown in Fig. 9(a) is similar to that of CdSe superlattices except that there is a manifold of four bands instead of one in CdSe. Like in $\mathrm{CdSe}$, these bands are mainly formed by the coupling $\left(V_{s s \sigma}\right)$ between $1 S$ conduction wave functions of nearest neighbor $\mathrm{PbSe}$ NCs. The four $1 S \mathrm{NC}$ wave functions originate from the fourfold-degenerate $\mathrm{CB}$ of bulk $\mathrm{PbSe}$ at the $L$ point of the Brillouin zone (eightfold degeneracy including the spin), the situation being symmetric in the VB. ${ }^{24,27}$ Higher energy CBs and lower energy $\mathrm{VBs}$ are due to the $1 P$ and $1 D \mathrm{NC}$ wave functions (only four bands are shown in Fig. 9 for clarity). The evident splitting between all the $1 S(1 P)$ bands indicates that the energy and coupling of $\mathrm{NC}$ wave functions are not only defined by the symmetry of the envelope function $1 S(1 P)$ but also by the underlying Bloch function which depends on the originating valley (intervalley splitting ${ }^{24}$ ). The absence of translational symmetry due to the reduced dimensionality in the square superlattices induces coupling between states which originate from the different $L$ valleys, whereas they are degenerate in bulk PbSe. The effect of intervalley coupling has been discussed in Refs. 24 and 28 for PbSe NCs, and has been extensively investigated in the case of $\mathrm{Si}$ nanostructures and $\mathrm{Si} / \mathrm{Ge}$ heterostructures ${ }^{34,44-49}$ where an oscillating behavior of the intervalley splitting was found with well width, $\mathrm{NC}$ size.

The importance of valley effects on the band structure of the $\mathrm{PbSe}$ superlattices is also demonstrated when we compare Figs. 9(b) and 9(d) with Figs. 9(a) and 9(c) corresponding to two different $\mathrm{NC}$ sizes. We have found that, depending on the number $n$ of $\{100\}$ biplanes, being either an even or odd number, the position of the CB minimum and VB maximum alternates between $\Gamma$ and $M$. In fact, the $1 S$ bands both in the $\mathrm{CB}$ and $\mathrm{VB}$ are completely reversed between the two situations, meaning that the sign of $V_{s s \sigma}$ changes with the
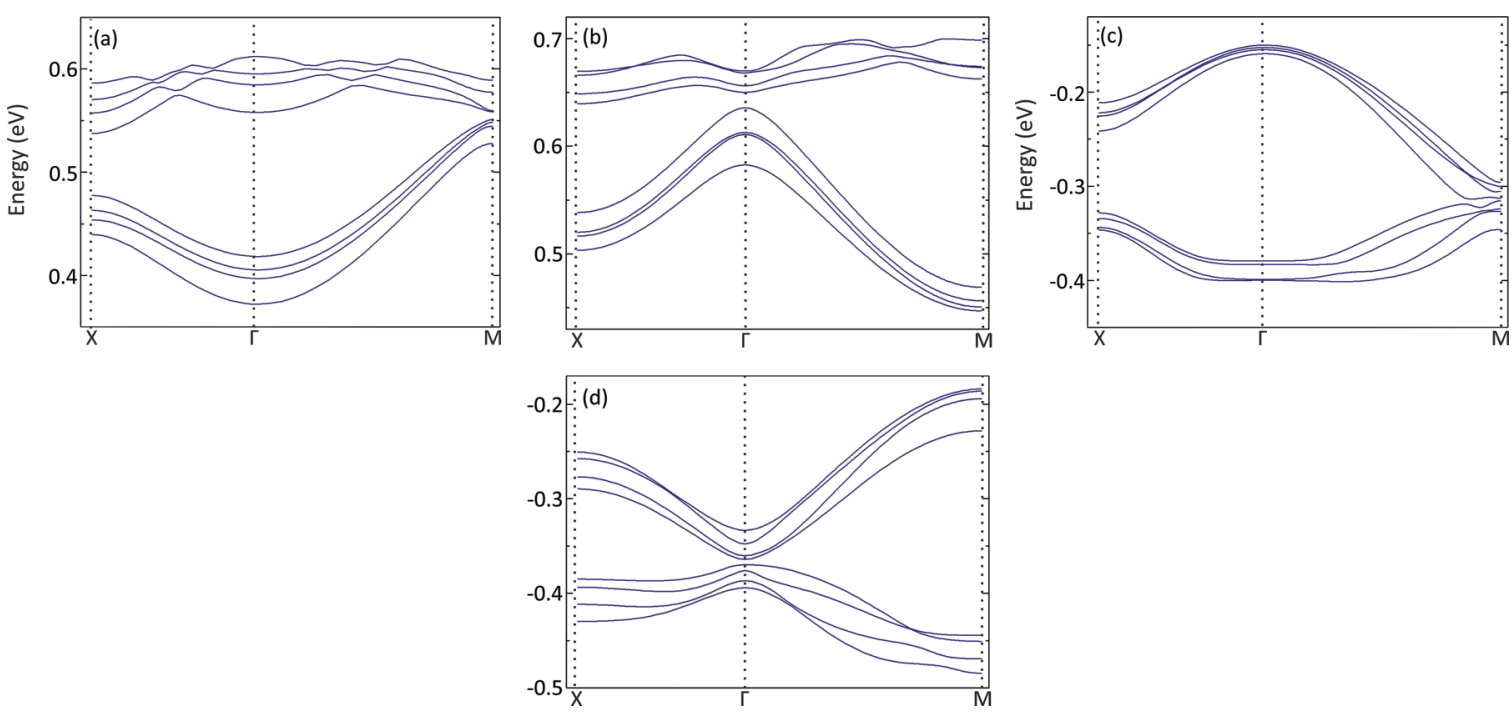

FIG. 9. (Color online) (a) CB and (c) VB structure for a superlattice of $4.89 \mathrm{~nm} \mathrm{PbSe} \mathrm{NCs}(n=8, q=0.45)$. (c) and (d) Corresponding plots for $4.28 \mathrm{~nm} \mathrm{NCs}(n=7, q=0.45)$. 
parity of $n$. This can be elucidated by considering two atoms positioned at a distance $n a$ along a $\langle 100\rangle$ direction. The phase of the Bloch function on the two atoms at the $L$ point of the Brillouin zone (at $\mathbf{k}_{L}$ ) is alternating for odd and even $n$ as $\exp \left[i \mathbf{k}_{L} \cdot(n a, 0,0)\right] \propto \cos (\pi n)$. This unusual behavior cannot take place in superlattices of semiconductors with band edges at $\Gamma$ and could be exploited for band engineering in superlattices of $\mathrm{PbSe}$. Besides this surprising feature, the qualitative characteristics of the bands are the same for odd and even $n$.

\section{CONCLUSION}

In summary, we have investigated the electronic structure in square superlattices of rock-salt $\mathrm{PbSe}$ or zinc-blende $\mathrm{CdSe}$ (CdTe) NCs attached via $\{100\}$ facets. We have used a $s p^{3} d^{5} s^{\star}$ tight-binding method, which allows the examination of $\mathrm{NC}$ sizes accessible to experiment. In all cases, a rich electronic structure consisting of multiple dispersive bands is observed, which can be directly attributed to the nanogeometry imposed to the $2 \mathrm{D}$ crystalline sheets. The number of atoms at the neighboring NCs bonding plane, defined by their shape and size, is established as the critical parameter determining the width of the bands. These planar systems form a new class of semiconductor materials with a dimensionality that decreases gradually below two if the effects of the nanogeometry, i.e., the periodic array of holes, becomes more important. Still, a significant dispersion of both conduction and valence bands is predicted for all compounds, promising efficient bandlike transport of electrons and holes. This is unlike carrier transport in nanocrystal superlattices in which the nanocrystals are separated by an organic or inorganic barrier, resulting in a much weaker coupling. Our results designate square superlattices as a highly attractive template for photodetectors, solar cells, and field-effect transistors. Our calculations further reveal large spin splitting in the electronic structure of square superlattices of CdTe NCs. In the case of PbSe superlattices, the position in $k$ space of the conduction and valence band edges depends on whether the number of biplanes of atoms in the NCs is odd or even. These results should stimulate further work on the topic including theoretical and experimental investigation of the transport properties of these systems. In particular, local scanning tunneling microscopy and spectroscopy should give important information on their electronic structure.

\section{ACKNOWLEDGMENTS}

This work has been supported by funding of the French National Research Agency [ANR, (ANR-09-BLAN-0421-01)]. E.K., C.D., and G.A. performed the calculations. W.H.E performed sample preparation and structural analysis. C.D. was a visiting professor at the Debye Institute for Nanomaterials Science at the time of this research. C.D. and D.V. supervised the project. All authors were involved in writing of the paper.

\section{APPENDIX A: TIGHT-BINDING PARAMETERS}

We consider a double basis of $s p^{3} d^{5} s^{\star}$ atomic orbitals for each $\mathrm{Pb}, \mathrm{Cd}, \mathrm{Se}$, or Te atom, including the spin degree of
TABLE I. Tight-binding parameters (notations of Slater and Koster ${ }^{37}$ ) for zinc-blende CdSe in an orthogonal $s p^{3} d^{5} s^{\star}$ model. $\Delta$ is the spin-orbit coupling. (a) and (c) denote the anion (Se) and the cation $(\mathrm{Cd})$, respectively.

\begin{tabular}{lrcr}
\hline \hline & \multicolumn{3}{c}{ Parameters for CdSe $(\mathrm{eV})$} \\
\hline$E_{s}(\mathrm{a})$ & -8.065657 & $E_{s}(\mathrm{c})$ & -1.857148 \\
$E_{p}(\mathrm{a})$ & 4.870028 & $E_{p}(\mathrm{c})$ & 5.613460 \\
$E_{d_{x y}}(\mathrm{a})$ & 15.671502 & $E_{d_{x y}}(\mathrm{c})$ & 16.715749 \\
$E_{d_{x^{2}-y^{2}}}(\mathrm{a})$ & 15.232107 & $E_{d_{x^{2}-y^{2}}}(\mathrm{c})$ & 20.151047 \\
$E_{s^{*}}(\mathrm{a})$ & 15.636238 & $E_{s^{*}}(\mathrm{c})$ & 20.004452 \\
$\Delta(\mathrm{a})$ & 0.140000 & $\Delta(\mathrm{c})$ & 0.150000 \\
$V_{s s \sigma}(\mathrm{ac})$ & -1.639722 & $V_{s^{*} s^{*} \sigma}(\mathrm{ac})$ & -1.805116 \\
$V_{s s^{*} \sigma}(\mathrm{ac})$ & 1.317093 & $V_{s s^{*} \sigma}(\mathrm{ca})$ & 0.039842 \\
$V_{s p \sigma}(\mathrm{ac})$ & 3.668731 & $V_{s p \sigma}(\mathrm{ca})$ & 1.885956 \\
$V_{s^{*} p \sigma}(\mathrm{ac})$ & 0.978722 & $V_{s^{*} p \sigma}(\mathrm{ca})$ & 1.424094 \\
$V_{s d \sigma}(\mathrm{ac})$ & -0.890315 & $V_{s d \sigma}(\mathrm{ca})$ & -1.007270 \\
$V_{s^{*} d \sigma}(\mathrm{ac})$ & 0.906630 & $V_{s^{*} d \sigma}(\mathrm{ca})$ & 2.472941 \\
$V_{p p \sigma}(\mathrm{ac})$ & 4.430196 & $V_{p p \pi}(\mathrm{ac})$ & -0.798156 \\
$V_{p d \sigma}(\mathrm{ac})$ & -2.645560 & $V_{p d \sigma}(\mathrm{ca})$ & -1.296749 \\
$V_{p d \pi}(\mathrm{ac})$ & 0.028089 & $V_{p d \pi}(\mathrm{ca})$ & 2.295717 \\
$V_{d d \sigma}(\mathrm{ac})$ & -2.480060 & $V_{d d \pi}(\mathrm{ac})$ & 2.393224 \\
$V_{d d \delta}$ & -1.373199 & & \\
& Parameters for Cd-H and Se-H (eV) & \\
$E_{H}$ & 0.000000 & & 61.82948 \\
$V_{s s \sigma}$ & -35.69727 & $V_{s p \sigma}$ & \\
\hline \hline
\end{tabular}

freedom. For $\mathrm{PbSe}$, we use the tight-binding parameters of Ref. 24. Due to the lack of $s p^{3} d^{5} s^{\star}$ tight-binding parameters for zinc-blende $\mathrm{CdSe}$ and $\mathrm{CdTe}$, corresponding data were derived and are presented in Tables I and II, respectively. They were

TABLE II. Same as Table I but for zinc-blende CdTe. These parameters have already been used in Ref. 18 .

\begin{tabular}{lccr}
\hline \hline \multicolumn{4}{c}{ Parameters for CdTe $(\mathrm{eV})$} \\
\hline$E_{s}(\mathrm{a})$ & -8.716293 & $E_{s}(\mathrm{c})$ & -1.269161 \\
$E_{p}(\mathrm{a})$ & 2.362764 & $E_{p}(\mathrm{c})$ & 5.739082 \\
$E_{d_{x y}}(\mathrm{a})$ & 11.204600 & $E_{d_{x y}}(\mathrm{c})$ & 15.107061 \\
$E_{d_{x^{2}-y^{2}}}(\mathrm{a})$ & 13.061473 & $E_{d_{x^{2}-y^{2}}}(\mathrm{c})$ & 17.014361 \\
$E_{s^{*}}(\mathrm{a})$ & 13.802893 & $E_{s^{*}}(\mathrm{c})$ & 17.908140 \\
$\Delta(\mathrm{a})$ & 0.385000 & $\Delta(\mathrm{c})$ & 0.065000 \\
$V_{s s \sigma}(\mathrm{ac})$ & -1.372451 & $V_{s^{*} s^{*} \sigma}(\mathrm{ac})$ & -1.768994 \\
$V_{s s^{*} \sigma}(\mathrm{ac})$ & 0.177593 & $V_{s s^{*} \sigma}(\mathrm{ca})$ & 0.171087 \\
$V_{s p \sigma}(\mathrm{ac})$ & 2.464283 & $V_{s p \sigma}(\mathrm{ca})$ & 2.010538 \\
$V_{s^{*} p \sigma}(\mathrm{ac})$ & 0.299617 & $V_{s^{*} p \sigma}(\mathrm{ca})$ & 0.299793 \\
$V_{s d \sigma}(\mathrm{ac})$ & -0.999978 & $V_{s d \sigma}(\mathrm{ca})$ & -0.947854 \\
$V_{s^{*} d \sigma}(\mathrm{ac})$ & -0.231393 & $V_{s^{*} d \sigma}(\mathrm{ca})$ & -0.047237 \\
$V_{p p \sigma}(\mathrm{ac})$ & 3.633352 & $V_{p p \pi}(\mathrm{ac})$ & -0.770868 \\
$V_{p d \sigma}(\mathrm{ac})$ & -0.994358 & $V_{p d \sigma}(\mathrm{ca})$ & -0.166481 \\
$V_{p d \pi}(\mathrm{ac})$ & 1.364603 & $V_{p d \pi}(\mathrm{ca})$ & 2.200983 \\
$V_{d d \sigma}(\mathrm{ac})$ & -2.100122 & $V_{d d \pi}(\mathrm{ac})$ & 1.714043 \\
$V_{d d \delta}$ & -0.727556 & & \\
& Parameters for Cd-H and Te-H (eV) & \\
$E_{H}$ & 0.000000 & & 61.82948 \\
$V_{s s \sigma}$ & -35.69727 & $V_{s p \sigma}$ & \\
\hline \hline
\end{tabular}


obtained by fitting the experimental effective masses and a reference band structure at the main points of the Brillouin zone and in a large energy range. The reference band structure was calculated using the ab initio pseudopotential code ABINIT $^{50}$ in the local density approximation. The pseudopotentials of Ref. 51 were used. The gap was corrected using a scissor operator to fit with its experimental value.

\section{APPENDIX B: EFFECTIVE EMPIRICAL PSEUDOPOTENTIAL MODEL}

In this Appendix we show that we can recover the band structure of the square superlattices starting from the limit of the uniform film $(q=0)$ if we introduce pseudopotentials which describe the effect of the periodic truncation. We consider for simplicity the case of the CB characterized in the bulk by an effective mass $m^{\star}$. In the uniform film, minibands are formed due to the confinement. Their energy dispersion is given by $E_{n}^{\mathrm{c}}+\hbar^{2} k^{2} /\left(2 m^{\star}\right)$, where $E_{n}^{\mathrm{c}}$ is the bottom of the miniband $n$ and $k$ is the modulus of the in-plane wave vector $\mathbf{k}$. We write $\mathbf{k}=\mathbf{K}+\mathbf{G}$, where $\mathbf{K}$ is the wave vector in the reduced Brillouin zone of the superlattice and $\mathbf{G}$ is a reciprocal lattice vector $[\mathbf{G}=(i, j) 2 \pi / L$, where $L$ is the length of the square supercell, and $i$ and $j$ are integers]. The eigenvalues and eigenstates of the Hamiltonian $H_{0}$ for the uniform film are given, respectively, by

$$
\begin{gathered}
E_{n, \mathbf{G}}(\mathbf{K})=E_{n}^{\mathrm{c}}+\frac{\hbar^{2}|\mathbf{K}+\mathbf{G}|^{2}}{2 m^{\star}}, \\
\Psi_{n, \mathbf{G}}(\mathbf{K})=\chi_{n}(z) \exp \left[i(\mathbf{K}+\mathbf{G}) \cdot \mathbf{r}_{\|}\right],
\end{gathered}
$$

where $\mathbf{r}_{\|}$is the in-plane position vector and $\chi_{n}(z)$ is the wave function along the transverse direction $z$. Figure 10(a) shows that Eq. (B1) reproduces quite well the results of the tight-binding calculations of Fig. 3(a).

The potential $V$ induced by the truncation $(q \neq 0)$ on the electrons is periodic and therefore can be written in Fourier series as

$$
V\left(\mathbf{r}_{\|}, z\right)=\sum_{\mathbf{G}} V(\mathbf{G}, z) \exp \left[i \mathbf{G} \cdot \mathbf{r}_{\|}\right] .
$$

We deduce that the matrix elements of $V$ in the basis of the eigenstates for the uniform film [Eq. (B2)] can be simplified into

$$
\left\langle\Psi_{n, \mathbf{G}}(\mathbf{K})|V| \Psi_{n^{\prime}, \mathbf{G}^{\prime}}(\mathbf{K})\right\rangle=V_{n, n^{\prime}}\left[\left|\mathbf{G}-\mathbf{G}^{\prime}\right|\right]
$$
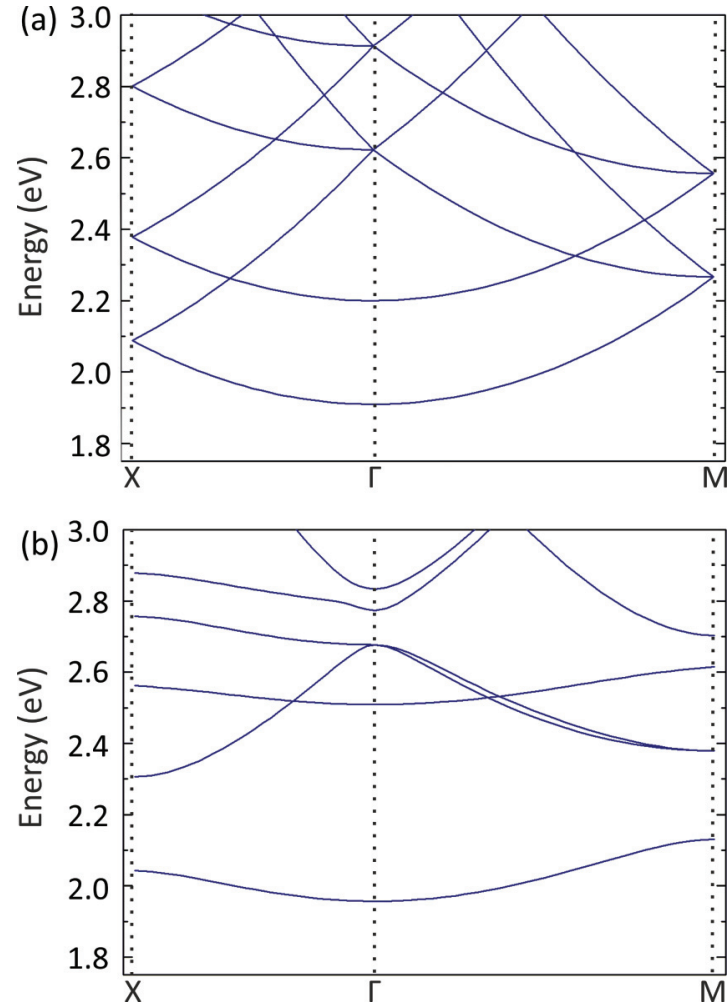

FIG. 10. (Color online) (a) Conduction band structure of a uniform film of CdSe assuming free-electron dispersion of Eq. (B1) $\left(m^{\star}=0.1 m_{0}, E_{1}^{\mathrm{c}}=1.91 \mathrm{eV}, E_{2}^{\mathrm{c}}=2.20 \mathrm{eV}, L=4.53 \mathrm{~nm}\right)$. (b) Same but for a square superlattice, including pseudopotential terms describing the scattering of electrons induced by the periodic truncations: $V_{1,1}[0]=0.15 \mathrm{eV}, V_{1,1}[2 \pi / L]=0.15 \mathrm{eV}, V_{1,1}[2 \sqrt{2} \pi / L]=0.02 \mathrm{eV}$, $V_{1,1}[4 \pi / L]=0.04 \mathrm{eV}, V_{2,2}[0]=0.70 \mathrm{eV}, V_{2,2}[2 \pi / L]=0.35 \mathrm{eV}$, $V_{2,2}[2 \sqrt{2} \pi / L]=0.08 \mathrm{eV}, V_{2,2}[4 \pi / L]=0.08 \mathrm{eV}$, where the quantity in the brackets denotes the value of $\left|\mathbf{G}-\mathbf{G}^{\prime}\right|$.

which only depend on $n, n^{\prime}$ and $\left|\mathbf{G}-\mathbf{G}^{\prime}\right|$. In addition, by symmetry, $V$ only couples bands $n$ and $n^{\prime}$ with the same parity. If we look at the low-energy part of the band structure, we can write the matrix of $H_{0}+V$ in a limited basis of states $\Psi_{n, \mathbf{G}}(\mathbf{K})$ (e.g., $n=1,2$ ) and the terms $V_{n, n^{\prime}}\left[\left|\mathbf{G}-\mathbf{G}^{\prime}\right|\right]$ can be seen as parameters. Figure 10 shows that we can obtain a band structure behaving as in Fig. 3(b) for reasonable values of these parameters. A similar pseudopotential approach could be developed for holes using for example a $\mathbf{k} \cdot \mathbf{p}$ Hamiltonian.

\footnotetext{
*christophe.delerue@isen.fr

${ }^{1}$ K. Barnham and D. Vvedensky (eds.), Low Dimensional Semiconductor Structures: Fundamentals and Applications (Cambridge University Press, Cambridge, 2001).

${ }^{2}$ K. S. Novoselov, A. K. Geim, S. V. Morozov, D. Jiang, Y. Zhang, S. V. Dubonos, I. V. Grigorieva, and A. A. Firsov, Science 306, 666 (2004).

${ }^{3}$ B. Yan and S.-C. Zhang, Rep. Prog. Phys. 75, 096501 (2012).

${ }^{4}$ M. Z. Hasan and C. L. Kane, Rev. Mod. Phys. 82, 3045 (2010).

${ }^{5}$ M. Henini (ed.), Molecular Beam Epitaxy: From Research to Mass Production (Elsevier, New York, 2001).
}

${ }^{6}$ S. Ithurria and B. Dubertret, J. Am. Chem. Soc. 130, 16504 (2008).

${ }^{7}$ S. Ithurria, M. D. Tessier, B. Mahler, R. P. S. M. Lobo, B. Dubertret, and A. L. Efros, Nat. Mater. 10, 936 (2011).

${ }^{8}$ W. H. Evers, B. Goris, S. Bals, M. Casavola, J. de Graaf, R. v. Roij, M. Dijkstra, and D. Vanmaekelbergh, Nano Lett. 13, 2317 (2013).

${ }^{9}$ W. J. Baumgardner, K. Whitham, and T. Hanrath, Nano Lett. 13, 3225 (2013).

${ }^{10}$ E. Kalesaki, C. Delerue, C. Morais Smith, G. Allan, and D. Vanmaekelbergh (unpublished).

${ }^{11}$ D. V. Talapin, J.-S. Lee, M. V. Kovalenko, and E. V. Shevchenko, Chem. Rev. 110, 389 (2010). 
${ }^{12}$ M. V. Kovalenko, M. Scheele, and D. V. Talapin, Science 324, 1417 (2009).

${ }^{13}$ E. Talgorn, R. D. Abellon, P. J. Kooyman, J. Piris, T. J. Savenije, A. Goossens, A. J. Houtepen, and L. D. A. Siebbeles, ACS Nano 4, 1723 (2010).

${ }^{14}$ D. Vanmaekelbergh and P. Liljeroth, Chem. Soc. Rev. 34, 299 (2005).

${ }^{15}$ D. Steiner, D. Azulay, A. Aharoni, A. Salant, U. Banin, and O. Millo, Nanotechnology 19, 065201 (2008).

${ }^{16}$ D.-K. Ko, J. J. Urban, and C. B. Murray, Nano Lett. 10, 1842 (2010).

${ }^{17}$ The Landolt-Börnstein Database, Semiconductors: NonTetrahedrally Bonded Binary Compounds II (Springer, Berlin, 2000), Vol. 41D.

${ }^{18}$ E. Groeneveld, C. Delerue, G. Allan, Y.-M. Niquet, and C. de Mello Donegá, J. Phys. Chem. C 116, 23160 (2012).

${ }^{19}$ C. de Mello Donegá, M. Bode, and A. Meijerink, Phys. Rev. B 74, 085320 (2006).

${ }^{20}$ G. D. Scholes, Adv. Funct. Mater. 18, 1157 (2008).

${ }^{21}$ A. Franceschetti, H. Fu, L. W. Wang, and A. Zunger, Phys. Rev. B 60, 1819 (1999).

${ }^{22}$ P. E. Lippens and M. Lannoo, Phys. Rev. B 41, 6079 (1990).

${ }^{23}$ A. L. Efros, M. Rosen, M. Kuno, M. Nirmal, D. J. Norris, and M. Bawendi, Phys. Rev. B 54, 4843 (1996).

${ }^{24}$ G. Allan and C. Delerue, Phys. Rev. B 70, 245321 (2004).

${ }^{25}$ R. Koole, G. Allan, C. Delerue, A. Meijerink, D. Vanmaekelbergh, and A. Houtepen, Small 4, 127 (2008).

${ }^{26}$ A. N. Poddubny, M. O. Nestoklon, and S. V. Goupalov, Phys. Rev. B 86, 035324 (2012).

${ }^{27}$ I. Kang and F. W. Wise, J. Opt. Soc. Am. B 14, 1632 (1997).

${ }^{28}$ J. M. An, A. Franceschetti, S. V. Dudiy, and A. Zunger, Nano Lett. 6, 2728 (2006).

${ }^{29}$ R. J. Ellingson, M. C. Beard, J. C. Johnson, P. Yu, O. I. Micic, A. J. Nozik, A. Shabaev, and A. L. Efros, Nano Lett. 5, 865 (2005).

${ }^{30}$ W.-K. Koh, A. C. Bartnik, F. W. Wise, and C. B. Murray, J. Am. Chem. Soc. 132, 3909 (2010).

${ }^{31}$ F. W. Wise, Acc. Chem. Res. 33, 773 (2000).

${ }^{32}$ H. Du, C. Chen, R. Krishnan, T. D. Krauss, J. M. Harbold, F. W. Wise, M. G. Thomas, and J. Silcox, Nano Lett. 2, 1321 (2002).

${ }^{33} \mathrm{C}$. Delerue and M. Lannoo, Nanostructures: Theory and Modeling (Springer, Berlin, 2004).
${ }^{34}$ Y. M. Niquet, C. Delerue, G. Allan, and M. Lannoo, Phys. Rev. B 62, 5109 (2000).

${ }^{35}$ G. Allan, G. Bastard, N. Boccara, M. Lannoo, and M. Voos (eds.), Heterojunctions and Semiconductor Superlattices: Proceedings of the Winter School Les Houches, France, March 12-21, 1985 (Springer, Berlin, 1986).

${ }^{36}$ W. A. Harrison, Electronic Structure and the Properties of Solids: The Physics of the Chemical Bond (Dover, New York, 1989).

${ }^{37}$ J. C. Slater and G. F. Koster, Phys. Rev. 94, 1498 (1954).

${ }^{38}$ G. Dresselhaus, Phys. Rev. 100, 580 (1955).

${ }^{39}$ E. Rashba and E. Sherman, Phys. Lett. A 129, 175 (1988).

${ }^{40}$ H. Mayer and U. Rössler, Solid State Commun. 87, 81 (1993).

${ }^{41}$ I. Žutić, J. Fabian, and S. Das Sarma, Rev. Mod. Phys. 76, 323 (2004).

${ }^{42}$ M. I. D'yakonov and V. Y. Kachorovskii, Fiz. Tekh. Poluprovodn. 20, 178 (1986) [Sov. Phys. Semicond. 20, 110 (1986)].

${ }^{43}$ N. S. Averkiev, L. E. Golub, and M. Willander, J. Phys.: Condens. Matter 14, R271 (2002).

${ }^{44}$ D. Z.-Y. Ting and Y.-C. Chang, Phys. Rev. B 38, 3414 (1988).

${ }^{45}$ G. Allan, C. Delerue, and Y. M. Niquet, Phys. Rev. B 63, 205301 (2001).

${ }^{46}$ T. B. Boykin, G. Klimeck, M. Friesen, S. N. Coppersmith, P. von Allmen, F. Oyafuso, and S. Lee, Phys. Rev. B 70, 165325 (2004).

${ }^{47}$ T. B. Boykin, G. Klimeck, P. von Allmen, S. Lee, and F. Oyafuso, J. Appl. Phys. 97, 113702 (2005).

${ }^{48}$ M. Friesen, S. Chutia, C. Tahan, and S. N. Coppersmith, Phys. Rev. B 75, 115318 (2007).

${ }^{49}$ M. O. Nestoklon, L. E. Golub, and E. L. Ivchenko, Phys. Rev. B 73, 235334 (2006).

${ }^{50}$ X. Gonze, B. Amadon, P.-M. Anglade, J.-M. Beuken, F. Bottin, P. Boulanger, F. Bruneval, D. Caliste, R. Caracas, M. Côté, T. Deutsch, L. Genovese, P. Ghosez, M. Giantomassi, S. Goedecker, D. R. Hamann, P. Hermet, F. Jollet, G. Jomard, S. Leroux, M. Mancini, S. Mazevet, M. J. T. Oliveira, G. Onida, Y. Pouillon, T. Rangel, G.-M. Rignanese, D. Sangalli, R. Shaltaf, M. Torrent, M. J. Verstraete, G. Zerah, and J. W. Zwanziger, Comput. Phys. Commun. 180, 2582 (2009).

${ }^{51}$ C. Hartwigsen, S. Goedecker, and J. Hutter, Phys. Rev. B 58, 3641 (1998). 